Santa Clara University

Scholar Commons

Electrical Engineering

School of Engineering

4-28-2008

\title{
Length dependence of current-induced breakdown in carbon nanofiber interconnects
}

Hirohiko Kitsuki

Toshishige Yamada

SantaClara University, tyamada@scu.edu

Drazen Fabris

SantaClara University, dfabris@scu.edu

John R. Jameson

Patrick Wilhite

Santa Clara University, pwilhite@scu.edu

See next page for additional authors

Follow this and additional works at: https://scholarcommons.scu.edu/elec

\section{Recommended Citation}

H. Kitsuki, T. Yamada, D. Fabris, J.R. Jameson, P. Wilhite, M. Suzuki, and C.Y. Yang, "Length dependence of current-induced breakdown in carbon nanofiber interconnects," Applied Physics Letters 92, 173110 (3 pp) (2008). https://doi.org/10.1063/ 1.2918839

Copyright (C 2008 American Institute of Physics Publishing. Reprinted with permission.

This Article is brought to you for free and open access by the School of Engineering at Scholar Commons. It has been accepted for inclusion in

Electrical Engineering by an authorized administrator of Scholar Commons. For more information, please contact rscroggin@scu.edu. 
Authors

Hirohiko Kitsuki, Toshishige Yamada, Drazen Fabris, John R. Jameson, Patrick Wilhite, Makoto Suzuki, and Cary Y. Yang 


\title{
Length dependence of current-induced breakdown in carbon nanofiber interconnects
}

\author{
Hirohiko Kitsuki, Toshishige Yamada, a) Drazen Fabris, John R. Jameson, Patrick Wilhite, \\ Makoto Suzuki, and Cary Y. Yang \\ Center for Nanostructures, Santa Clara University, Santa Clara, California 95053, USA
}

(Received 14 March 2008; accepted 10 April 2008; published online 1 May 2008)

\begin{abstract}
Current-induced breakdown is investigated for carbon nanofibers (CNF) for potential interconnect applications. The measured maximum current density in the suspended CNF is inversely proportional to the nanofiber length and is independent of diameter. This relationship can be described with a heat transport model that takes into account Joule heating and heat diffusion along the CNF, assuming that breakdown occurs when and where the temperature reaches a threshold or critical value. () 2008 American Institute of Physics. [DOI: 10.1063/1.2918839]
\end{abstract}

Because of their high electrical and thermal conductivities as well as current capacity, carbon nanotubes ${ }^{1}$ (CNTs) and carbon nanofibers ${ }^{2-5}$ (CNFs) are being investigated for high-performance device and interconnect applications. Breakdown phenomena have been observed under highcurrent stress conditions for $\mathrm{CNTs}^{6-12}$ and amorphous carbon nanowires. ${ }^{13}$ Breakdown mechanisms generally depend on the detailed carbon nanostructures. For CNFs, recent studies $^{5,14,15}$ suggest current-induced CNF breakdown to be closely related to Joule heating, and thermal coupling between CNF and electrodes affects the maximum current density $J_{\max }$ of CNF devices and their breakdown. Meanwhile, current annealing has been reported to drastically reduce the overall resistance and this may be attributed to significant lowering of the contact resistances in CNT devices. ${ }^{16-18}$ Therefore, one can assume that once the contact resistances are reduced, the reliability of CNF interconnects becomes largely dependent on the nanofiber resistance. In this work, systematic characterization of CNFs under high-current stress is performed to examine and to elucidate the CNF breakdown phenomena.

The CNF samples are grown using plasma-enhanced chemical vapor deposition ${ }^{19,20}$ with a $\mathrm{Ni}$ catalyst layer on a Si substrate. A 30-nm-thick Ti adhesion layer is used between the 35-nm-thick $\mathrm{Ni}$ layer and $\mathrm{Si}$, and a gas mixture of $\mathrm{NH}_{3}: \mathrm{C}_{2} \mathrm{H}_{2}$ (4:1) at 4 Torr is used for the reaction. ${ }^{21} \mathrm{~A}$ solution of CNFs is drop-casted onto a substrate of prepatterned gold electrodes on an oxidized silicon wafer. The structure shown in Fig. 1(a) is a model of an on-chip interconnect configuration, where the CNF sidewall is in contact with the electrodes. ${ }^{22}$ Constant-current stress or anneal is applied in ambient [Fig. 1(b)] for 13 samples, ranging from 100 to $200 \mathrm{~nm}$ in diameter and 1.5 to $6 \mu \mathrm{m}$ in length.

The progression of constant-current stress cycles (at $180 \mathrm{~s}$ each) is illustrated in Fig. 2(a). At the end of each cycle, $I-V$ characteristics are obtained around $V=0$. Increasing the annealing current results in a gradual decrease in the differential resistance $R$ at $V=0$ before the nanofiber breaks down at $700 \mu \mathrm{A}$ [Fig. 2(b)]. Since $R$ consists of bulk and contact contributions, and the CNF consistently breaks near the middle away from the contacts, current annealing likely reduces the contact resistances significantly, while break-

a) The author to whom correspondence should be addressed. Electronic mail:
tyamada@scu.edu. down occurs due to Joule heating in the CNF bulk.

Systematic analysis using scanning transmission electron microscopy (STEM) and in situ scanning electron microscopy (SEM) measurements has revealed the creation of void and defective graphitic layers in the CNF induced by current stress. ${ }^{14}$ This analysis suggests that the CNF resistance just before breakdown drastically increases due to severe degradation of the nanofiber internal structure. The SEM image of a CNF before current stress is shown in Fig. 3(a). In all experiments for suspended CNFs, we have confirmed that breakdown always occurs near the middle of the nanofiber as in Figs. 3(b) and 3(c). This is consistent with diffusive heat transport in CNTs at high bias, ${ }^{10}$ suggesting the importance of Joule heating ${ }^{15}$ in breakdown.

To understand these experimental results, a onedimensional (1D) thermal transport model ${ }^{15,23}$ is used. We define $\Delta T(x)$ as the difference between the local temperature $T(x)$ and the temperature at infinity. $\Delta T(x)$ is determined from the balance among heat diffusion $\left(d^{2} \Delta T / d x^{2}\right)$, heat dissipation to the surroundings $\left(a^{2} \Delta T\right)$, and heat generation due to Joule heating $(f)$ in

$$
\frac{d^{2} \Delta T}{d x^{2}}-a^{2} \Delta T=-f
$$

Here, $f=I^{2} /\left(A^{2} \sigma \kappa\right)$, where $I$ is the current, $A$ is the crosssectional area, $\sigma$ is the electrical conductivity, and $\kappa$ is the CNF thermal conductivity. Also, $a^{2}=w \gamma / A \kappa$, where $w$ is the effective contact line width and $\gamma$ is the coupling coefficient for heat transport to the CNF surroundings (air and

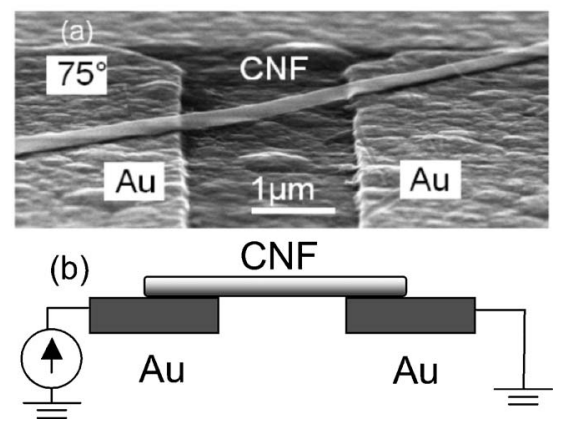

FIG. 1. Setup for current-stressing experiments. (a) SEM image of a CNF sample suspended between gold electrodes at $75^{\circ}$ tilted-angle view. Highresolution STEM images of CNFs can be seen in Ref. 14. (b) Schematic of electrical measurement. 

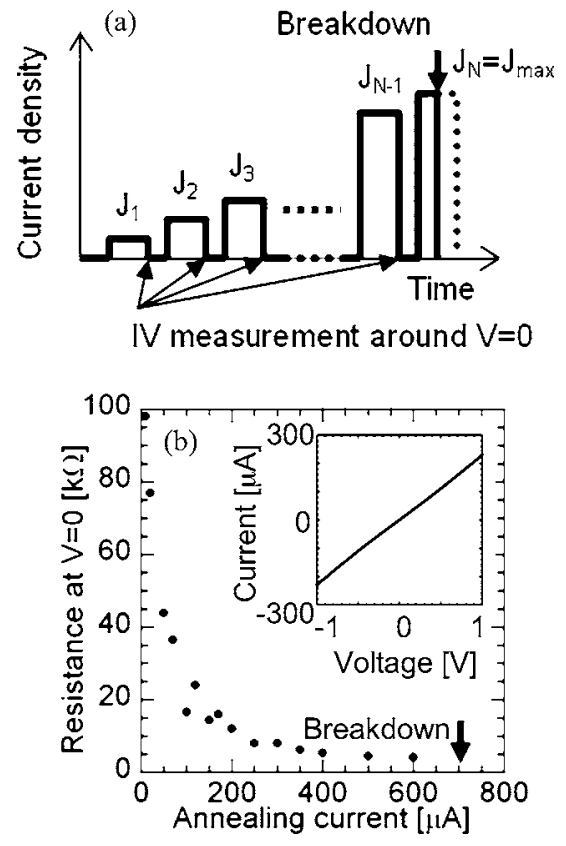

FIG. 2. Resistance reduction of CNF device due to current annealing. (a) Schematic of successive current annealing cycles using stepwise increment of stressing current. (b) Resistance of the CNF device at $V=0$ obtained after each annealing cycle. The inset shows the current-voltage behavior at the end of one of the anneal cycles.

substrate). We impose $\Delta T=0$ at the two ends where $x= \pm L / 2$ and $x=0$ is the midpoint. Thus, $\Delta T(x)=\left(f / a^{2}\right)[1$ $-\cosh (a x) / \cosh (a L / 2)]$ results, with the maximum at $x=0$. We assume that, in general, breakdown occurs when $T$ reaches a threshold (critical) temperature $T_{\max }$, which is common for all CNFs with any diameters or lengths, prepared using the same growth process. Then, the maximum current density $J_{\max }$ can be expressed as

$$
J_{\max }=\left[\left(T_{\max }-T_{\infty}\right) \sigma \gamma w / A\right]^{1 / 2}[1-1 / \cosh (a L / 2)]^{-1 / 2} .
$$

In the suspended case, heat dissipation is expected to be negligible, or $a L \ll 1$, yielding $J_{\max } \approx 2 \sqrt{2\left(T_{\max }-T_{\infty}\right) \sigma \kappa / L}$.

Figure 4(a) shows the experimental results (dots) for $J_{\max }$ versus $1 / L$ for 13 devices. The behavior is consistent with current-induced breakdown in single-walled CNTs ${ }^{24}$ or gold nanowires fabricated using conventional lithography techniques. ${ }^{25}$ The same behavior is predicted in our model in
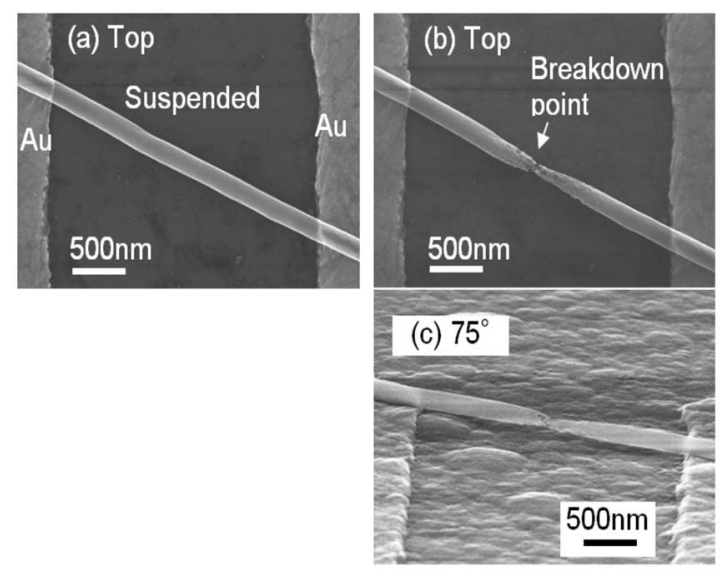

FIG. 3. (a) SEM images of a CNF suspended gold electrode before current stressing. (b) CNF after breakdown (top view) and (c) at $75^{\circ}$ tilted-angle view.
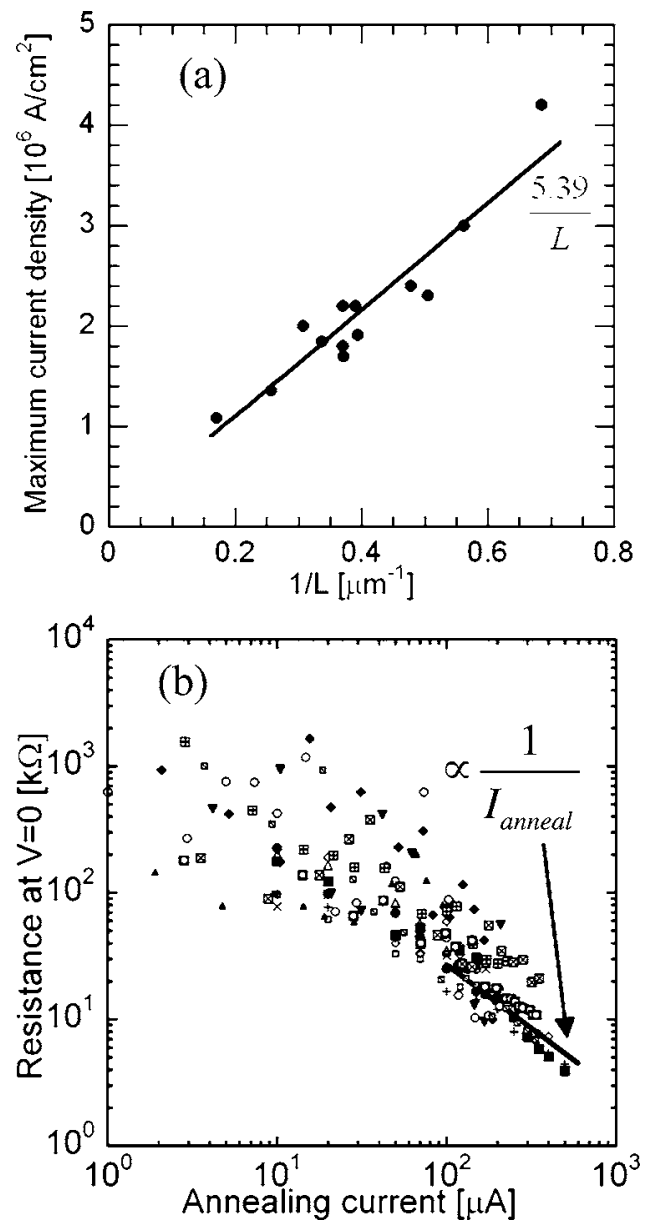

FIG. 4. (a) Maximum current density as a function of reciprocal CNF length for thirteen devices. The line shows a linear fit predicted by our heat transport model. (b) Resistance of CNF device as a function of annealing current after each annealing cycle for the same thirteen devices. The line approximates the resistance just before breakdown.

the $a L \ll 1$ limit of Eq. (2). This excellent agreement confirms that for a suspended CNF, heat dissipation to its surroundings is, in fact, small and heat diffusion along the CNF leads to the highest temperature in the middle of the nanofiber. Moreover, $J_{\max }$ scales with $1 / L$, which is consistent with the peak current behavior of suspended single-walled CNTs. $^{24}$

From the fitted result of $J_{\max }=5.39 / L$ in Fig. 4(a), $T_{\max }$ $=1260 \mathrm{~K}$. This is comparable to the CNF synthesis temperature, estimated to be in the $1000 \mathrm{~K}$ range. Here, a CNF thermal conductivity of $12 \mathrm{~W} / \mathrm{m} \mathrm{K}^{26}$ and the maximum electrical conductivity from the present data are used. Having no radiative heat transfer and in the limit of no coupling with the substrate, the temperature estimate is only an indication of CNF durability and points to the need for systematic local temperature measurement.

Our previous work showed a $J_{\max } \propto 1 / \sqrt{L}$ behavior, ${ }^{14}$ based on the assumption that CNF was a long 1D thermal conductor with an exponential decay of temperature beyond the CNF/electrode contacts. In the present work, the heat dissipation to the substrate is weak over the suspended distance, while the nanofiber coupling with the electrodes is relatively strong, where $\Delta T=0$ is assumed. The difference in boundary conditions leads to $J_{\max }$ scaling with $1 / L$ as verified by the present experimental results. 
Figure 4(b) shows a plot of $R$ measured at the end of each current annealing cycle as a function of annealing current $I_{\text {anneal }}$ for all 13 devices. We note that each CNF has a different diameter and length, with different contact properties. For this reason, one can expect that well before breakdown when $J_{\max }$ is reached, $R$ significantly deviates from sample to sample partly due to large contact resistances, up to a factor of 20 in our case. However, after successive current annealing cycles as illustrated in Fig. 2(a), $R$ begins to converge for $I_{\text {anneal }}>100 \mu \mathrm{A}$, and the deviation from sample to sample is reduced to less than a factor of 5 .

This result can be understood by assuming that the contact resistance is initially large and dominates $R$ but is significantly reduced through repeating current annealing, and for $I_{\text {anneal }}>100 \mu \mathrm{A}$, the bulk CNF resistance dominates. In fact, during these later annealing cycles, long CNFs have already broken down, as shown in Fig. 4(a) and the surviving CNFs are mostly short or less than $2.5 \mu \mathrm{m}$. For smaller $L$ and negligible contact resistance, the dominant source for resistance variation is the cross-sectional area $A$. Since the CNF diameter ranges from 100 to $200 \mathrm{~nm}, A$ varies up to a factor of 4 and $R$ is also expected to vary accordingly, which is consistent with the observed high- $I_{\text {anneal }}$ behavior in Fig. 4(b).

In Fig. 4(b), for samples with $I_{\text {anneal }}>100 \mu \mathrm{A}$, the resistance just before breakdown converges to $R \propto 1 / I_{\text {anneal }}$, regardless of $A$ and $L$ values. Assuming an Ohmic behavior, we have $R=L \rho / A=J_{\text {anneal }} L \rho / I_{\text {anneal }}$, where $\rho$ is the resistivity. In the final annealing cycle, $J_{\text {anneal }} \sim J_{\max }$. Since we have established that $J_{\max } \propto 1 / L, R \propto 1 / I_{\text {anneal }}$ results, confirming the empirical findings in Fig. 4(b).

In conclusion, current-induced breakdown has been investigated for suspended CNFs. With reduced contact resistance due to current annealing, a simple relationship between the maximum current capacity and CNF length is obtained experimentally and successfully explained with a 1D heat transport model. This result suggests that breakdown occurs when the local temperature reaches a geometry-independent value at the midpoint of the suspended nanofiber. The observed $J_{\max } \propto 1 / L$ behavior is consistent with metallic nanowires, and the present study represents an important step toward understanding the reliability of CNF for potential interconnect applications.

We are grateful to J. Li and A. M. Cassell for their helpful advice and to Hitachi High-Technologies America for its assistance in electron microscopy. This work was supported by the United States Army Space and Missile Defense Command (SMDC) and carries Distribution Statement A, approved for public release, distribution unlimited.
${ }^{1}$ See, e.g., Carbon Nanotubes: Synthesis, Structure, Properties, and Applications, edited by R. E. Smalley, M. S. Dresselhaus, G. Dresselhaus, and $\mathrm{Ph}$. Avouris (Springer, Boston, 2001).

${ }^{2}$ L. C. Qin, D. Zhou, A. R. Kraus, and D. M. Gruen, Appl. Phys. Lett. 72, 3437 (1998).

${ }^{3}$ J. Li, Q. Ye, A. M. Cassell, H. T. Ng, R. Stevens, J. Han, and M. Meyyappan, Appl. Phys. Lett. 82, 2491 (2003).

${ }^{4}$ F. Kreupl, A. P. Graham, M. Liebau, G. S. Duesberg, R. Seidel, and E. Unger, Tech. Dig. - Int. Electron Devices Meet. 2004, 683.

${ }^{5}$ Q. Ngo, A. M. Cassell, A. J. Austin, J. Li, S. Krishnan, M. Meyyappan, and C. Y. Yang, IEEE Electron Device Lett. 27, 221 (2006).

${ }^{6}$ A. Javey, J. Guo, M. Paulsson, Q. Wang, D. Mann, M. Lundstrom, and H. Dai, Phys. Rev. Lett. 92, 106804 (2004).

${ }^{7}$ E. Pop, D. A. Mann, J. Cao, K. E. Goodson, and H. Dai, J. Appl. Phys. 101, 093710 (2007).

${ }^{8}$ P. G. Collins, M. Hersam, M. Arnold, R. Martel, and Ph. Avouris, Phys. Rev. Lett. 86, 3128 (2001).

${ }^{9}$ B. Bourlon, D. C. Glattli, B. Plaçais, J. M. Berroir, C. Miko, L. Forró, and A. Bachtold, Phys. Rev. Lett. 92, 026804 (2004).

${ }^{10}$ J. Y. Huang, S. Chen, S. H. Jo, Z. Wang, D. X. Han, G. Chen, M. S. Dresselhaus, and Z. F. Ren, Phys. Rev. Lett. 94, 236802 (2005).

${ }^{11}$ T. D. Yuzvinsky, W. Mickelson, S. Aloni, S. L. Konsek, A. M. Fennimore, G. E. Begtrup, A. Kis, B. C. Regan, and A. Zettl, Appl. Phys. Lett. 87, 083103 (2005).

${ }^{12}$ M. S. Wang, J. Y. Wang, Q. Chen, and L.-M. Peng, Adv. Funct. Mater. 15, 1825 (2005).

${ }^{13}$ C. H. Jin, J. Y. Wang, Q. Chen, and L.-M. Peng, J. Phys. Chem. B 110, 5423 (2006).

${ }^{14}$ M. Suzuki, Y. Ominami, Q. Ngo, C. Y. Yang, J. Li, and A. M. Cassell, J. Appl. Phys. 101, 114307 (2007).

${ }^{15}$ M. A. Kuroda, A. Cangellaris, and J.-P. Leburton, Phys. Rev. Lett. 95, 266803 (2005).

${ }^{16} \mathrm{Q}$. Ngo, "Electrical and Thermal Characterization of Carbon Nanofibers for Interconnect and Packaging Applications," Ph.D. thesis, Santa Clara University, 2006

${ }^{17}$ J.-O. Lee, C. Park, J.-J. Kim, J. Kim, J. W. Park, and K.-H. Yoo, J. Phys. D 33, 1953 (2000).

${ }^{18}$ E. Minoux, O. Groening, K. B. K. Teo, S. H. Dalal, L. Gangloff, J.-P. Shnell, I. Y. Y. Bu, P. Vincent, P. Legagneux, G. A. J. Amaratunga, and W. I. Milne, Nano Lett. 5, 2135 (2005).

${ }^{19}$ Y. Chen, Z. L. Wang, J. S. Yin, D. J. Johnson, and R. H. Prince, Chem. Phys. Lett. 272, 178 (1997).

${ }^{20}$ Z. F. Ren, Z. P. Huang, J. W. Xu, J. H. Wang, P. Bush, M. P. Siegal, and P. N. Provencio, Science 282, 1105 (1998).

${ }^{21}$ B. A. Cruden, A. M. Cassell, Q. Ye, and M. Meyyappan, J. Appl. Phys. 94, 4070 (2003).

${ }^{22}$ L. Zhang, D. Austin, V. I. Merkulov, A. V. Meleshko, K. L. Klein, M. A. Guillorn, D. H. Lowndes, and M. L. Simpson, Appl. Phys. Lett. 84, 3972 (2004).

${ }^{23}$ H. S. Carslaw and J. C. Jaeger, Conduction of Heat in Solids, 2nd ed. (Oxford, Oxford, 1986).

${ }^{24}$ E. Pop, D. Mann, J. Cao, Q. Wang, K. Goodson, and H. Dai, Phys. Rev. Lett. 95, 155505 (2005).

${ }^{25}$ C. Durkan, M. A. Schneider, and M. E. Welland, J. Appl. Phys. 86, 1280 (1999).

${ }^{26}$ C. Yu, S. Saha, J. Zhou, L. Shi, A. M. Cassell, B. A. Cruden, Q. Ngo, and J. Li, J. Heat Transfer 128, 234 (2006). 